\title{
Preferences on Online Gambling Activities among Adolescents: A Review
}

González-Bueso V1,*,†, Santamaría JJ ${ }^{1, *, \dagger}$, Fernández $D^{2,3}$, Montero $E^{1}$, Baño $M^{1}$, J iménezMurcia $\mathrm{S}^{4,5,6}$, del Pino-Gutiérrez $\mathrm{A}^{4,5,7}$ and Ribas J ${ }^{1}$ ${ }^{1}$ Attention and Research in Socioaddictions (AIS), Mental Health and Addictions Network, Spain

2Serra Húnter Fellow, Department of Statistics and Operations Research, Polytechnic University of CataloniaBarcelonaTech, 08028 Barcelona, Spain

${ }^{3}$ Center for Biomedical Research in Mental Health Network (CIBERSAM), Spain

${ }^{4}$ Department of Psychiatry, Bellvitge University HospitalIDIBELL, Spain

${ }^{5}$ CIBER Pathophysiology Obesity and Nutrition (CIBER), Carlos III Health Institute, Spain

${ }^{6}$ Department of Clinical Sciences, University of Barcelona, Spain

7Department of Public Health, Mental Health and

Perinatal Nursing, School of Nursing, University of

Barcelona-UB, Barcelona, Spain

†These authors contributed equally to this work and share first authorship

*Corresponding author: Gonzalez-Bueso V

and Santamaria JJ , Attention and Research in

Socioaddictions (AIS), Mental Health and Addictions

Network, Government of Catalonia (SISCAT), 08014

Barcelona, Spain

Received: J anuary 13, 2021; Accepted: February 11, 2021; Published: February 18, 2021

\begin{abstract}
The accessibility and availability of a variety of online gambling for adolescents is a public concern. In the current literature, it remains unclear in which activities the greatest prevalence occurs. Moreover, it is well-known that different types of gambling activities carry different levels of risk just as have distinct socioeconomic, and mental health-related consequences. The main aim of this study is to systematically review the current literature in order to explore the prevalence of different types of online gambling activities reported by adolescents and their relationship with other reported variables when available. It will be conducted following the Preferred Reporting Items for Systematic Reviews and Meta-Analyses-P 2015 statement for systematic review and metaanalysis protocols. An electronic literature search will be conducted using the following databases: PubMed, PsychINFO, ScienceDirect, Web of Science, and Google Scholar using search keywords and logic as follows: "(Internet OR online) gambling AND (adolescents OR young people)". Additionally, further articles will be identified through searching the citations in the literature selected. The overall percentage of online gamblers and the percentage of online gamblers for each type of gambling activity were identified or calculated. Seven-teen articles met the eligibility criteria. The included studies comprised 15 crosssectional and two longitudinal designs. Most of the research was conducted in Europe. The online gambling modalities detected were sports bets, card games, gambling machines, casino games lottery games, scratch tickets, bingo, gambling in social networking, stock market investment, and mahjong. Only two studies provided associations between a specific online gambling activity and socioeconomic and mental health associations, founding involvement in online sports betting and in online casino game is a risk factor to the development of disordered gambling. A consensus on the evaluation method of the problem and updated questionnaires collecting information regarding the new online betting modalities are critical.
\end{abstract}

Keywords: Online Gambling Activities; Adolescents; Review; Mental Health; Prevalence

\section{Introduction}

Nowadays, land-based and online gambling is an relevant global mental health issue, that causes health and social problems to the addicted individuals and those who lose the behavior control, affecting all their areas of life, including comorbid mental health, family, financial, and legal issues [1-3].

The inherent characteristics of the different types of gambling activities and the aspects of the gambling environment are associated with different levels of gambling problem severity and comorbid harms [4]. Regarding the gambling mode, high event frequency, intermittent sizes of rewards, and fast play seem to lead to higher severity [5]. Regarding the environment, some of the most problematic factors are accessibility, high immersive ambiance, the ability to obtain additional money, easy access to multiple gambling activities, and the possibility to continue playing without interruptions [6-8].

Taking into account the different gambling activities in a similar way, the type of gambling preference can vary depending on demographics $[9,10]$ and also can have an influence on the severity and other psychosocial problems. Several authors found differences in gender, age, income, or ethnic, as well as high rates of problem gambling, differences in substance use, psychological distress, psychiatric problems depending on the problematic gambling type [11-14].

Some authors argue that online gambling is only a modality of playing the same presential games of chance, and not a game by itself [15]. However, few differences have been reported between both modalities regarding its characteristics. Online gambling seems to possess various differential characteristics not or less present in land-based modalities, that coincide with the aspects associated with problem severity [16]. First of all, access to gambling sites is effortless and present-day technologies allow to enter gambling sites any time of the day, from any location, via any platform connected to the Internet such as smartphones, tablets, interactive televisions, and laptops. This characteristic is commonly associated with motivations to play $[17,18]$. Moreover, the stimuli affecting the gambling behavior of the customers can be adapted and personalized instantaneously, based on the characteristics of each gambler, with the aim of
J Psychiatry Mental Disord - Volume 6 Issue 1 - 2021

Submit your Manuscript | www.austinpublishing group.com Gonzalez-Bueso et al. @ All rights are reserved
Citation: Gonzalez-Bueso V, Santamaria JJ, Fernandez D, Montero E, Bano M, Jimenez-Murcia S, et al. Preferences on Online Gambling Activities among Adolescents: A Review. J Psychiatry Mental Disord. 2021; 6(1): 1033. 
achieving frequent or even excessive play behavior. Other specific and unique characteristics of the internet gambling leading to gambling problems are the possibility to access immediately to a variety of gambling games, including the option to play many different games concurrently, and the quick event response where the player can vary or select faster gambling modes [19-21].

At present, there are more opportunities for adolescents to start and carry on gambling due to the quick-change nature of the digital technologies, where new types of games, technologies to access them, and gambling providers have proliferated fast [22]. In spite of gambling is an age-restricted activity in most of the countries, several authors found that this activity (online) is relatively common in adolescents and is also increasingly recognized as a major public health problem [22-24], although in a percentage less common than the land-based variant [25-28]. Regarding prevalence, the current research has found that it varies between $0.2 \%$ and $12.3 \%$ in adolescents, showing values of 2.5-5.6\% of adolescents who meet risk gambling criteria [29-31].

Gambling during the adolescence is associated with a variety of mental health symptoms (e.g., anxiety, depression, or low selfesteem), negative behaviors such as the use of alcohol and drugs $[23,32-34]$, and other unfavorable effects such as conflicting family relationships, criminal acts [35], increased ideation or suicide attempts and/or other addictions [24] and poor general health [36].

Despite these studies identified adolescent gambling, including Internet gambling, little is known about the adolescent preferences with respect to online gambling forms and about the mental health and socioeconomic consequences of each of these modalities.

The aim of this study is to review systematically the current literature to explore the different types of online gambling activities reported by adolescents and, as a secondary objective, the associations found with other reported variables. An additional objective is to analyze the prevalence of online gambling and of each online gambling modalities in adolescents. Such results can furnish clinicians with updated information and provide a direction for future research. The objective of this paper is not to analyze problematic adolescent online gamblers, but to focus on analyzing their preferences when it comes to gambling online.

\section{Material and Methods}

The present review was conducted following the Preferred Reporting Items for Systematic Reviews and Meta-Analyses-P 2015 statement for systematic review and meta-analysis protocols [20]. From April 2020 to May 2020 we reviewed the following databases: PubMed, ScienceDirect, and Google Scholar, using search keywords and logic as follows: "(Internet OR online) gambling AND (adolescents OR young people)”.

To avoid confusion between the different online activities that may or may not be considered as gambling online, the particular activity was assessed as internet gambling when it was an online game where the outcome is predominately determined by chance, in which payment is required, and where monetary prizes may be awarded [37]. We defined online gambler user as adolescents that had gambled online at least one time during the previous 12 months.

These database search filters gave more than 35.500 results.
Specifically, each database yield the following results: PubMed (58 results), and ScienceDirect (639 results). The search in Google Scholar returned more than 35.100 results. In consequence, we reviewed the first 45 pages of results. Additional articles ( 2 results), were identified through the citations in the literature selected. The first authors (Vega Gonzalez-Bueso and Juan Jose Santamaria) systematically and independently reviewed the studies. The variables take into account were the study type, methodology, study population, outcome measures, prevalence, and interpretation of results. The discordances were resolved through accords or referral to a third reviewer (Daniel Fernandez). The applied inclusion criteria were: (I) empirically collected data about Online Gambling (OG); (II) the OG was assessed by standardized questionnaires, diagnostic criteria based on international disease classifications or tools developed to detect most commonly reported gambling symptoms; (III) the different gambling activities were assessed and results about each modality were informed; (IV) mental health comorbidity assessed by standardized questionnaires and/or sociodemographic variables related to OG were assessed; (V) participants aged between 11 and 20 years; (VI) full text available; (VII) data of publication after 2010 (in order to review the most recent research in a field where the subject evolves rapidly); (VIII) English or Spanish language (the two languages known by the authors); and (IX) article published in a peer-reviewed journal.

The exclusion criteria were: (I) articles using only anecdotal evidence; (II) case series or case reports; (III) studies only reporting results on phenomenon such as motivation to gamble, decisionmaking, lifestyle, impulsivity, without reporting another psychiatric comorbidity or sociodemographic variables; and (IV) studies only reposting results on online gambling as a unique activity.

If there were studies in which the sample analyzed had age ranges different from 11-20 years, then they were also included only if the results were shown broken down according to age groups and there was a specified age range between 11 and 20 years. Additionally, if there were studies in which the object of research was land-based gambling, then they were also included only if different online-based activities were specified and the results were broken down according to both methods. The geographical distribution of studies was mapped (Table 1). When possible, the overall percentage of online gamblers and the percentage of online gamblers for each type of gambling activity with respect to the whole sample were identified or calculated.

As our research work is a review, the mean prevalences reported were computed within the articles in a range of different ways. Generally speaking, we calculated sample-size-weighted mean prevalences when the sample size information was available or there was a way to obtain it making direct computations from the results or data sets reported in the article. For example, if an article did not report the raw sample size, but the percentages of the total sample were reported, then we made the appropriate computations to obtain the sample sizes used later in the mean. All the prevalences reported in our work are calculated based on sample-size-weighted mean prevalence's.

\section{Results}

A total of 151 studies were identified and screened trough the systematic search protocol, after discard duplicated articles. The application of the inclusion and exclusion criteria left a total of 17 
studies which were included in the review. Summary of the main characteristics of the articles reporting different types of online gambling activities by adolescents, including prevalence for each gambling type when possible (Table 1). When the authors did not clearly specify the activity by online or land-based, it was removed from the results.

\section{Design of the included studies}

Fifteen of the 17 articles included were cross-sectional studies [30,38,47-51,39-46], the two remaining articles were longitudinal studies $[52,53]$. In descending order, the countries/regions were the research was performed were Spain (2), Australia (2), Canada (2), Hong Kong (2), United Kingdom (2), Denmark (1), France (1), Italy (1), Norway (1), and Romania (1), Turkey (1) and USA (1). Most of the studies (59\%) were performed in Europe.

\section{Characteristics of the used samples}

The total sample used in the 17 studies was 77,852 participants. All studies examined both genders. The range of participants in each study was from 322 to 14,778 ( $M=4325.11$; Standard Deviation (SD): 4336.93). The age range between the studies was from 11 to 20 years. All seventeen studies were conducted in the general population, most of the studies were done in the school setting $[29,30,48-51,53,38,41$ 47], one was performed in a clinical setting [52].

\section{Methods of assessing Online Gambling Disorder (OGD)}

Most of the studies $[30,41,46,49,53]$ used the South Oaks Gambling Screen-Revised for Adolescents SOGS-RA [54], which is a 12 dichotomous items questionnaire, and one 4-levels item. The scores return three categories: non-problem gambler, at-risk gambler, and problem gambler.

Since 2013, the DSM-5 includes diagnostic criteria for Gambling Disorder [55], the second most used questionnaire [40,44,50] was the DSM-IV-Multiple Response-Juvenile (DSM-IV-MR-J) [56]. This instrument is based on the DSM-IV diagnostic criteria for Pathological Gambling [57], which contains nine items assessing progression and preoccupation, tolerance, withdrawal, and loss of control. The maximum and minimum scores are 9 and 0 , respectively. It classifies participants as social gamblers, at-risk gamblers, and problem gamblers.

Two of the included articles $[42,48]$ used the Canadian Adolescent Gambling Inventory (CAGI) [58]. This inventory consists of nine items assessing behaviors with psychological, social, financial, and inhibitory consequences, which are related to gambling in the past three months. Gambling severity scores were then categorized as no problem gambling, low to moderate gambling severity, and high gambling severity.

The remaining studies applied the Canadian Problem Gambling Index [59], the 20 questions of the Gamblers Anonymous American Association [60], and the DSM-IV Screen for Gambling ProblemsLoss of Control, Lying, and Preoccupation (NODS-CLiP) [61]. All authors used ad-hoc surveys intending to identifying online gambling activities and other gambling-related variables, which were not included in any of the selected questionnaires.

\section{Online gambling, gambling modalities and prevalence}

The mean prevalence estimates of different gambling activities found in the included articles (Table 1). The mean online gambling prevalence was $8.86 \%$ (SD: 7.19), with a minimum prevalence of $1.00 \%$ and a maximum prevalence of $28.90 \%$. Particularly, twelve articles detected gambling through sports bets $[29,30,49,50,38,39,41-$ $44,46,48]$, and card games [29,30,51,53,38,41,42,44,46,48-50], being the most frequent activities. Seven articles found online gambling machines [30,41,42,44,48,49,62] and casino games $[30,38,45,47,49,50,62]$, and six studies detected lottery games $[30,41,44,49,50,62]$.

The remaining gambling activities observed were (in order): scratch tickets in four articles $[30,41,44,49]$, bingo in three studies $[30,46,49]$, and gambling in social networking [29], stock market investment [43], and mahjong [50] in one article. Finally, Hollen [52] found online betting, defined as betting online on any event or sports, and online gaming, defined as playing poker, bingo, slot machinestyle games, or casino games for money online, in their sample.

Regarding of each gambling modality, not all authors provided enough data to identify or compute the percentage with respect to the whole sample. With the available information the most prevalent types of gambling, excluding those modalities only reported in one study (Table 1), were (in order of mean prevalence): sports bets (M $=7.82, \mathrm{SD}=9.75)$; card games $(\mathrm{M}=5.70, \mathrm{SD}=2.67)$; lottery $(\mathrm{M}=1.15$, $\mathrm{SD}=0.21)$; other casino games $(\mathrm{M}=0.98, \mathrm{SD}=1.06)$; online gambling machines $(\mathrm{M}=0.97, \mathrm{SD}=1.33)$; scratch tickets $(\mathrm{M}=0.55, \mathrm{SD}=0.07)$; and bingo $(\mathrm{M}=0.30, \mathrm{SD}=0.14)$. Only six studies gave results broken down by gender. Their outcomes indicate that the online gambling had a greater prevalence among males, having a mean prevalence of $\mathrm{M}=10.62$ ( $\mathrm{SD}=7.59)$, in contrast with the female mean prevalence of $\mathrm{M}=2.76(\mathrm{SD}=1.92)$.

Regarding gambling type prevalence according to gender, there were only two studies reporting this factor separately. Thus, the most common prevalent gambling modes for males were (in order): sports bets $(\mathrm{M}=6.75, \mathrm{SD}=7.56)$; card games $(\mathrm{M}=3.50, \mathrm{SD}=3.81)$; and online gambling machines $(\mathrm{M}=1.70, \mathrm{SD}=2.26)$. The same raking for females were as follows (in order): sports bets $(\mathrm{M}=1.45, \mathrm{SD}=1.46)$; electronic gambling machines $(\mathrm{M}=0.90, \mathrm{SD}=1.13)$; and card games $(\mathrm{M}=0.60$, $\mathrm{SD}=0.70)$.

\section{Socioeconomic and mental health associations}

Not all studies provided associations between online gambling and socioeconomic and mental health variables. Additionally, only two articles $[43,49]$ gave results regarding a specific gambling activity, which found that online sports betting and involvement in online casino games in the previous year is a risk factor to the development of disordered gambling. Several studies found that online gambling was more common in males [41,42,50,52]. Additionally, only a single study [49] reported that male gamblers were more likely to bet in mixed-mode venues than females. Nevertheless, mixed-mode gambling was related to gambling severity in females.

According to the majority of the results, the rates of problematic gambling, gambling severity, and higher risk for problem gambling were higher among online gamblers than offline gamblers $[41,42,46,50,62]$. In contrast, Gonzalez-Roz and colleagues [30] found that none of the online gamblers in their sample were problem gamblers, and also $10.2 \%$ of mixed-mode gamblers were problem gamblers. Online gamblers' engagement in more types of gambling 
Table 1: Studies reporting different types of online gambling activities by adolescents.

\begin{tabular}{|c|c|c|c|c|c|c|c|c|c|c|c|}
\hline Source & Study type & $\mathrm{N}$ & $\mathrm{Age}^{\mathrm{a}}$ & Sex & $\begin{array}{l}\text { Country/ } \\
\text { Region }\end{array}$ & OG Measures & Other Variables & $\%$ who OG ${ }^{b}$ & $\% \mathrm{PG}^{\mathrm{c}}$ & $\begin{array}{c}\text { Type of OG } \\
(\%)^{\mathrm{b}}\end{array}$ & Association \\
\hline $\begin{array}{l}\text { Lupu et al., } \\
2013 \text { [39] }\end{array}$ & $\begin{array}{l}\text { cross- } \\
\text { sectional }\end{array}$ & 1032 & $11-19$ & $\mathrm{M} / \mathrm{F}$ & Romania & $\begin{array}{c}\text { Gamblers } \\
\text { Anonymous } \\
\text { American } \\
\text { Association Test } \\
\text { ad-hoc Survey }\end{array}$ & $\begin{array}{l}\text { Sociodemographic } \\
\text { information }\end{array}$ & 1 & O/LB: 3.5 & $\begin{array}{c}\text { Internet casino } \\
\text { (1) }\end{array}$ & - \\
\hline $\begin{array}{l}\text { Rossow et } \\
\text { al., 2013 } \\
{[53]}\end{array}$ & Iongitudinal & $\begin{array}{c}4,912 \\
(2006) \\
3,855 \\
(2008)\end{array}$ & $13-18$ & $\mathrm{M} / \mathrm{F}$ & Norway & \begin{tabular}{|} 
South Oaks \\
Gambling Screen \\
-Revised for \\
Adolescents Lie/Bet \\
Questionnaire \\
ad-hoc Survey
\end{tabular} & $\begin{array}{l}\text { Sociodemographic } \\
\text { information Prevalence } \\
\text { trents }\end{array}$ & $\begin{array}{c}5.0(2006) 5.7 \\
\quad(2008)\end{array}$ & - & $\begin{array}{c}\text { Poker on } \\
\text { internet }(3.0) \\
(2006)(3.8) \\
(2008) \\
\text { Other internet } \\
\text { gambling(2.0) } \\
(2006)(1.9) \\
(2008)\end{array}$ & $\begin{array}{l}\text { Decrease in the weekly } \\
\text { gambling prevalence } \\
\text { over the years }\end{array}$ \\
\hline $\begin{array}{l}\text { Fu et al., } \\
2014 \text { [48] }\end{array}$ & $\begin{array}{l}\text { cross- } \\
\text { sectional }\end{array}$ & 700 & $17.0 \pm 1.25$ & $\mathrm{M} / \mathrm{F}$ & Hong Kong & $\begin{array}{c}\text { ad-hoc Survey (OG } \\
\text { Criteria based in } \\
\text { DSM 5) }\end{array}$ & $\begin{array}{l}\text { Sociodemographic } \\
\text { information } \\
\text { Internet gaming } \\
\text { addiction } \\
\text { Senses of control in } \\
\text { relation to gambling }\end{array}$ & $\mathrm{n} / \mathrm{a}$ & O/LB: 5.4 & $\begin{array}{l}\text { Online soccer } \\
\text { betting } \\
\text { Basketball } \\
\text { betting } \\
\text { (n/a) }\end{array}$ & $\begin{array}{l}\text { Sport betting is a risk } \\
\text { factor for disordered } \\
\text { gambling. }\end{array}$ \\
\hline $\begin{array}{l}\text { King et al., } \\
2014 \text { [49] }\end{array}$ & $\begin{array}{l}\text { cross- } \\
\text { sectional }\end{array}$ & 1,287 & $14.9 \pm 1.5$ & $\mathrm{M} / \mathrm{F}$ & Australia & $\begin{array}{l}\text { DSM-IV-Multiple } \\
\text { Response-Juvenile } \\
\text { (DSM-IV-MR-J) } \\
\text { ad-hoc Survey }\end{array}$ & $\begin{array}{l}\text { Sociodemographic } \\
\text { information } \\
\text { Anxiety and major } \\
\text { depressive symptoms }\end{array}$ & 2.3 & O/LB: 1.0 & \begin{tabular}{|} 
Card games \\
Electronic \\
gaming \\
machines \\
Sports bets \\
Lotteries \\
Scratch cards \\
Other activities \\
(n/a)
\end{tabular} & - \\
\hline $\begin{array}{l}\text { Kristiansen } \\
\text { et al., 2014 } \\
\text { [51] }\end{array}$ & $\begin{array}{l}\text { cross- } \\
\text { sectional }\end{array}$ & 2223 & $11-17$ & $\mathrm{M} / \mathrm{F}$ & Denmark & $\begin{array}{l}\text { South Oaks } \\
\text { Gambling Screen- } \\
\text { Revised for } \\
\text { Adolescents } \\
\text { ad-hoc Survey }\end{array}$ & 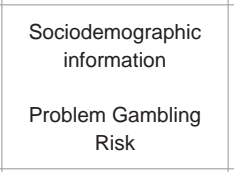 & 19.7 & O/LB: 1.2 & $\begin{array}{l}\text { Card games } \\
\text { Sports betting } \\
\text { Bingo (n/a) }\end{array}$ & $\begin{array}{c}\text { Significant } \\
\text { relationships between } \\
\text { mobile phone } \\
\text { gambling, online } \\
\text { gambling and gambling } \\
\text { severity }\end{array}$ \\
\hline $\begin{array}{l}\text { Wong et al., } \\
2014 \text { [42] }\end{array}$ & $\begin{array}{l}\text { cross- } \\
\text { sectional }\end{array}$ & 1,004 & $14.7 \pm 2.1$ & $\mathrm{M} / \mathrm{F}$ & Hong Kong & $\begin{array}{l}\text { DSM-IV-Multiple } \\
\text { Response-Juvenile } \\
\text { (DSM-IV-MRJ) } \\
\text { ad-hoc Survey }\end{array}$ & \begin{tabular}{|}
$\begin{array}{c}\text { Sociodemographic } \\
\text { variables }\end{array}$ \\
Internet usage \\
Perceptions of Internet \\
gambling activities
\end{tabular} & $\begin{array}{c}3.5 \\
\text { M: } 2.9 \\
\text { F: } 0.6\end{array}$ & O: 1.0 LB: 3.5 & $\begin{array}{l}\text { Mahjong } \\
\text { Poker cards } \\
\text { Casino dice } \\
\text { games } \\
\text { Soccer betting } \\
\text { Lotteries } \\
\text { Horse racing } \\
\text { (n/a) }\end{array}$ & $\begin{array}{c}\text { Rates of problematic } \\
\text { gambling are higher } \\
\text { among online gamblers } \\
\text { than offline gamblers. } \\
\text { Internet gambling is } \\
\text { a male-dominated } \\
\text { activity. }\end{array}$ \\
\hline $\begin{array}{l}\text { Canale et } \\
\text { al., 2016 } \\
{[46]}\end{array}$ & $\begin{array}{l}\text { cross- } \\
\text { sectional }\end{array}$ & 14,778 & $17.23 \pm 1.42$ & $\mathrm{M} / \mathrm{F}$ & Italy & $\begin{array}{c}\text { South Oaks } \\
\text { Gambling Screen- } \\
\text { Revised for } \\
\text { Adolescents ad-hoc } \\
\text { Survey }\end{array}$ & $\begin{array}{c}\text { Sociodemographic } \\
\text { information Internet for } \\
\text { leisure activities }\end{array}$ & $\begin{array}{c}15.6 \\
\text { M: } 11.6 \\
\text { F: } 2.4\end{array}$ & O: 3.4 LB: 0.6 & $\begin{array}{l}\text { Scratch tickets } \\
\text { Lottery Slot } \\
\text { machines and } \\
\text { VLT Sport } \\
\text { betting Other } \\
\text { events betting } \\
\text { Poker and } \\
\text { card games } \\
(\mathrm{n} / \mathrm{a})\end{array}$ & $\begin{array}{c}\text { Problem online } \\
\text { gamblers were more } \\
\text { likely to be male, to } \\
\text { live with two parents } \\
\text { and more likely to live } \\
\text { with others, spend } \\
\text { greater amounts } \\
\text { of money. Online } \\
\text { gamblers engaged } \\
\text { in significantly more } \\
\text { forms of gambling } \\
\text { compared with non- } \\
\text { online gamblers. Being } \\
\text { an online gambler was } \\
\text { positively associated } \\
\text { with adolescents' } \\
\text { problem gambling } \\
\text { severity }\end{array}$ \\
\hline $\begin{array}{l}\text { King et al., } \\
2016 \text { [50] }\end{array}$ & $\begin{array}{l}\text { cross- } \\
\text { sectional }\end{array}$ & 814 & $14.1 \pm 1.5$ & $\mathrm{M} / \mathrm{F}$ & Australia & ad-hoc Survey & $\begin{array}{l}\text { Sociodemographic } \\
\text { information Supervision } \\
\text { of gambling activities } \\
\text { Problem gambling } \\
\text { symptoms Parental } \\
\text { influences predicting }\end{array}$ & 4.9 & O/LB: 3.1 & $\begin{array}{l}\text { Online casino } \\
\text { (2.8) Mobile } \\
\text { app (2.1) }\end{array}$ & 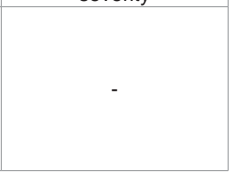 \\
\hline
\end{tabular}




\begin{tabular}{|c|c|c|c|c|c|c|c|c|c|c|c|}
\hline $\begin{array}{l}\text { Tara et al., } \\
2016[47]\end{array}$ & $\begin{array}{l}\text { cross- } \\
\text { sectional }\end{array}$ & 10,035 & $16.5 \pm 0.1$ & $\mathrm{M} / \mathrm{F}$ & Canada & $\begin{array}{l}\text { ad-hoc Survey } \\
\text { adapted from } \\
\text { the Canadian } \\
\text { Adolescent } \\
\text { Gambling Inventory }\end{array}$ & $\begin{array}{c}\text { Sociodemographic } \\
\text { variables }\end{array}$ & $\begin{array}{l}9.4 \\
\text { M: } 15.3 \\
\text { F: } 3.7\end{array}$ & O: 1.6 LB: 0.4 & $\begin{array}{c}\text { Internet poker } \\
(3.6) \text { Internet } \\
\text { poker }(3.6) \\
\text { M: } 6.2 \mathrm{~F}: 1.1 \\
\text { Sports pools } \\
\text { online }(7.3) \\
\text { M: } 12.1 \mathrm{~F}: 2.7 \\
\text { Slot machines } \\
\text { online }(2,5) \mathrm{M}: \\
\text { 3.3 F: } 1.7\end{array}$ & $\begin{array}{l}\text { Males who had } \\
\text { gambled were } \\
\text { significantly more } \\
\text { likely to gamble online } \\
\text { compared to females } \\
\text { who had gambled. } \\
\text { Online gamblers were } \\
\text { significantly more } \\
\text { likely to participate } \\
\text { in multiple gambling } \\
\text { modes compared to } \\
\text { land-based gamblers } \\
\text { online gamblers were } \\
\text { classified as "high" or } \\
\text { "low to moderate risk" } \\
\text { for problem gambling } \\
\text { compared to who } \\
\text { exclusively participated } \\
\text { in land-based } \\
\text { gambling }\end{array}$ \\
\hline $\begin{array}{l}\text { Baggio et } \\
\text { al., 2017 } \\
\text { [44] }\end{array}$ & $\begin{array}{l}\text { cross- } \\
\text { sectional }\end{array}$ & 9,910 & $17.4 \pm 0.01$ & $\mathrm{M} / \mathrm{F}$ & France & $\begin{array}{l}\text { Problem Gambling } \\
\text { Severity Index } \\
\text { (PGSI) ad-hoc } \\
\text { Survey }\end{array}$ & $\begin{array}{l}\text { Sociodemographic } \\
\text { information } \\
\text { Money spent gambling } \\
\text { Time spent gambling }\end{array}$ & $\begin{array}{c}10.5 \\
\text { M: } 7.3 \\
\text { F: } 3.2\end{array}$ & - & $\begin{array}{l}\text { Lottery Sport } \\
\text { betting and } \\
\text { pool games } \\
\text { Casino games } \\
\text { Gambling } \\
\text { machines } \\
\text { (n/a) }\end{array}$ & $\begin{array}{l}\text { Online gamblers } \\
\text { have higher levels of } \\
\text { disordered gambling, } \\
\text { spent more money } \\
\text { gambling, spent more } \\
\text { time gambling, and } \\
\text { reported a higher } \\
\text { diversity of gambling } \\
\text { formats than land- } \\
\text { based gamblers }\end{array}$ \\
\hline $\begin{array}{l}\text { Calado et } \\
\text { al., 2017 } \\
\text { [45] }\end{array}$ & $\begin{array}{l}\text { cross- } \\
\text { sectional }\end{array}$ & 988 & $19.8 \pm 2.0$ & $\mathrm{M} / \mathrm{F}$ & $\begin{array}{l}\text { United } \\
\text { Kingdom }\end{array}$ & $\begin{array}{l}\text { DSM-IV-Multiple } \\
\text { Response-Juvenile } \\
\text { (DSM-IV-MR-J) } \\
\text { ad-hoc Survey }\end{array}$ & $\begin{array}{l}\text { Sociodemographic } \\
\text { information } \\
\text { Gambling-related } \\
\text { cognitions Coping } \\
\text { styles } \\
\text { Attitudes toward } \\
\text { gambling } \\
\text { Perceptions of } \\
\text { relationship security }\end{array}$ & 28.9 & O/LB: 6.2 & $\begin{array}{c}\text { sports } \\
\text { betting (24.8) } \\
\text { Gambling } \\
\text { in social } \\
\text { networking } \\
\text { sites }(7.2) \\
\text { Blackjack (5.7) }\end{array}$ & \\
\hline $\begin{array}{l}\text { Gonzalez- } \\
\text { Roz et al., } \\
2017 \text { [30] }\end{array}$ & $\begin{array}{l}\text { cross- } \\
\text { sectional }\end{array}$ & 1,267 & $15.11 \pm 0.73$ & $\mathrm{M} / \mathrm{F}$ & Spain & $\begin{array}{l}\text { South Oaks } \\
\text { Gambling Screen- } \\
\text { Revised for } \\
\text { Adolescents } \\
\text { ad-hoc Survey }\end{array}$ & $\begin{array}{l}\text { Sociodemographic } \\
\text { information } \\
\text { Relatives with } \\
\text { gambling problems. }\end{array}$ & 9.1 & 3 & \begin{tabular}{|c|} 
Sports betting \\
$(2.8)$ Lottery \\
$(1.3)$ \\
Poker $(0.9)$, \\
Scratch tickets \\
$(0.6)$ \\
Bingo $(0.4)$ \\
Casino games \\
$(0.2)$ \\
Gambling \\
machines \\
$(0.2)$
\end{tabular} & $\begin{array}{c}10.2 \% \text { of mixed-mode } \\
\text { gamblers are problem } \\
\text { gamblers } \\
\text { None of the online } \\
\text { bettors were problem } \\
\text { gamblers }\end{array}$ \\
\hline $\begin{array}{l}\text { Veselka et } \\
\text { al., 2018 } \\
\text { [40] }\end{array}$ & $\begin{array}{l}\text { cross- } \\
\text { sectional }\end{array}$ & 10,035 & $13-19$ & $\mathrm{M} / \mathrm{F}$ & Canada & $\begin{array}{c}\text { Canadian } \\
\text { Adolescent } \\
\text { Gambling Inventory }\end{array}$ & $\begin{array}{c}\text { Sociodemographic } \\
\text { information Problem } \\
\text { gambling severity } \\
\text { Social casino gaming } \\
\text { Substance use } \\
\text { Screen time }\end{array}$ & $\begin{array}{l}1.9 \text { solely online } \\
7.6 \text { online and in } \\
\text { land-based }\end{array}$ & 2.2 & $\begin{array}{l}\text { Internet poker } \\
\text { Sports pools } \\
\text { Gambling } \\
\text { machines } \\
\text { (n/a) }\end{array}$ & $\begin{array}{c}\text { Associations between } \\
\text { social casino gaming } \\
\text { and problematic online } \\
\text { gambling }\end{array}$ \\
\hline $\begin{array}{c}\text { Weidberg } \\
\text { et al., 2018 } \\
\text { [41] }\end{array}$ & $\begin{array}{l}\text { cross- } \\
\text { sectional }\end{array}$ & 1756 & $15.22 \pm 0.74$ & $\mathrm{M} / \mathrm{F}$ & Spain & $\begin{array}{l}\text { South Oaks } \\
\text { Gambling Screen- } \\
\text { Revised for } \\
\text { Adolescents } \\
\text { ad-hoc Survey }\end{array}$ & $\begin{array}{l}\begin{array}{c}\text { Sociodemographic } \\
\text { information }\end{array} \\
\text { Gambling Motives } \\
\text { Impulsive Sensation- } \\
\text { Seeking Scale } \\
\text { Delay discounting task }\end{array}$ & $\begin{array}{c}4.7 \\
\text { M: } 3.8 \\
\text { F: } 0.9\end{array}$ & 5.7 & \begin{tabular}{|c|} 
Bingo $(0.2)$ \\
M:0.1, F:0.1 \\
Poker (1) \\
M:0.8, F:0.1 \\
Casino games \\
(0.3) M:0.3, \\
F:0.1 \\
Sports betting \\
(1.5) M:1.4, \\
F:0.2 \\
Lottery (1) \\
M:0.7, F:0.3 \\
Scratch-tickets \\
(0.5) M:0.3, \\
F:0.2 \\
Gambling \\
machines (0.2) \\
M:0.1, F:0.1
\end{tabular} & $\begin{array}{l}\text { Last year prevalence of } \\
\text { OCG was associated } \\
\text { with at risk/problem } \\
\text { gambling } \\
\text { Mixed-mode gambling } \\
\text { was related to } \\
\text { gambling severity in } \\
\text { females. } \\
\text { Male gamblers were } \\
\text { more likely to bet in } \\
\text { mixed-mode venues }\end{array}$ \\
\hline
\end{tabular}




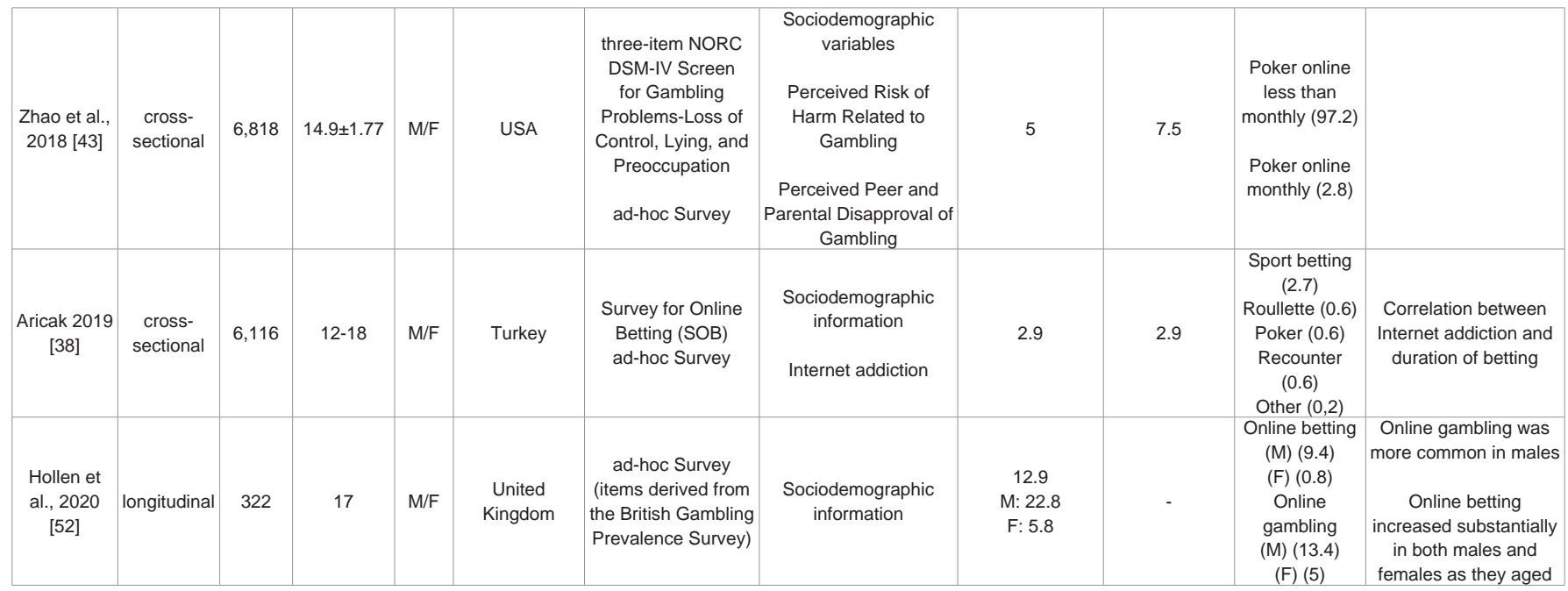

${ }^{a}$ Age is presented in years as a range or mean with SD. ${ }^{b}$ Male and Female percentage presented if possible. 'Percentage of problematic gambling reported. M/F: both males and females included. n/a: Non-enough data provided to calculate the mean or not applicable. O: Online Gaming; LD: Land-based gambling. O/LB: online and land based presented together.

compared with non-online gamblers was consistently reported across the selected studies $[41,42,62]$. Finally, regarding the prevalence of online gambling over the years, Hollen and colleagues [52] found that online betting increased in both males and females as they aged, and Rosow and colleagues [53] reported a decrease on weekly gambling prevalence over the years.

\section{Discussion}

The main purpose of this review was to explore the state of current literature about the preferred forms of online gambling activities in adolescents, its prevalence, and its relationships with other reported variables. We believe that this knowledge is crucial as both online gambling in young people is an increasing health problem and also different online gambling activities might be related to different problem severity and other psychosocial symptoms.

Although the specific gambling mode has influence and is relevant in the problem gambling severity $[5,63]$, some issues hinder the understanding of the online problem gambling in adolescents. Firstly, the measures used for problem gambling, i.e. the South Oaks Gambling Screen-Revised for Adolescents SOGS-RA [54], the DSMIV-Multiple Response-Juvenile (DSM-IV-MR-J) [56], the Canadian Adolescent Gambling Inventory (CAGI) [58], and the rest of the questionnaires set in the selected articles, do generally not distinguish between the different types of gambling modes. In the case they do, the types of games included are not updated to take into account the online modality, or the activities are not separated based on whether they are land-based or online-based. Thus, it forces the researchers and practitioners to develop ad-hoc surveys to measure this variable, generating consistency problems. Therefore, the online gambling preferences and the relationships between specific forms of gambling and socioeconomic and health associations in adolescents need to be interpreted carefully. Secondly, despite the studies analyzing online gambling preferences used large representative samples, most of them have been carried out in the general population in school settings, and only one study was focused on a clinical environment. This is a limitation that has been frequently highlighted in the literature of this area [64]. Studies are predominantly carried out via classroom surveys where is difficult to monitor the quality of the data collected. Thirdly, most of the studies selected in the first stage of this review, but excluded at the end, separated the different types of land-based gambling (i.e. poker, slot machines, bingo, etc.), but not the distinct modes of online gambling though. Thus, they considered it a sole modality and they, therefore, collected the data set or reported results as "internet gambling".

Regarding the prevalence of online gambling in adolescents, this behavior has been reported in all countries/regions with a prevalence mean around $9 \%$. Although this result is similar to that obtained in other studies [64], the range of values found in the reported studies was very large (from $1.00 \%$ to $29,80 \%$ ). It is difficult to determine if this variability may be due to several factors such as cultural or legal differences, or to the diversity of the measures applied, and the reference point used in each study when considering gambling behavior.

Regarding the assessment of the evolution of online gambling over the years, there are two longitudinal studies, which reported opposed results. Hollen et al., [52] found that online betting increased substantially in both sexes as they aged. However, Rosow et al., [53] reported a decrease in the gambling prevalence over the years. Hence, it is hard to conclude if online problem gambling among adolescents is stable or episodic. Again, the type of gambling activity may be influencing these inconsistent results as some authors have reported differences between different types of gamble and limited addictive power [65]. For both genders, lottery, scratch games, sports betting, and skill games seem to be less related to problem gambling [62] and, therefore, less associated to a stable activity over time.

In the reviewed papers specifying online gambling modes, the preferred modalities by the adolescent population are sports bets, card games, gambling machines, casino games, lottery games, scratch tickets, and bingo. Sports bets, card games, and lotteries were the modes with higher prevalence among them. However, the preferences are difficult to determine given that all studies used adhoc measures including or excluding gambling modalities according to each author's preference. 
Concerning gender differences, all the reviewed studies reported a higher prevalence of online gambling among males, which is similar to land-based gambling results [66]. Analyzing the gambling mode preferences in previous research, pure chance games (e.g. lottery, scratch games, and gambling machines) were common for both genders. Moreover, males preferred games involving perceived or actual skills such as sports betting and card games [67]. In contrast, in this review we found that males and females have a similar inclination for online sports bets, card games, and electronic gaming machines. We must take into account that only two studies reported the results about gambling modes broken down by gender, which makes any interpretation difficult.

Different gambling activities are associated with different levels of problem gambling, health problems, and worse treatment outcomes $[5,68]$. Additionally, it seems that there are several links between gender-specific gambling patterns and problem gambling such as the association between disordered gambling and gambling machines among females, and between sports betting, card, and casino games among males [62]. Therefore, we believe that this knowledge might be crucial for developing effective prevention and treatment interventions distinguishing between both sexes.

In this review, only two studies reported associations between specific online gambling activities and mental health variables. According to the reported results, the use of online sports bets and online casino games in the last year are risk factors to develop disordered gambling. According to Baggio and colleagues [62], sports betting and other skill-based casino games were less related to problem gambling. These ambiguous results show the complex relationship between age and gambling and highlight the need to take into account the differences between land-based and online gambling.

On the one hand, there is evidence suggesting that early onset of gambling behavior is a risk factor for developing problems with gambling $[69,70]$. Furthermore, the biological, personality, developmental, cognitive, and environmental factors associated with gambling behavior in adolescents [71] influence the game preferences or the consequences of each online modality. On the other hand, several studies in the adult population and land-based gambling have suggested that problem gambling is associated with high-frequency events, games promoting rapid gambling, and the immediacy of response [72].

In particular in the literature, Electronic Gaming Machines (EGMs) and online gambling in general, have shown to cause harm than other forms [73,74]. The inner and unique characteristics of the internet gambling make possible both to adapt all gambling modes to increase the gambling speed and to play easily many different games at the same time, increasing the event response and frequency, making the gambling activity more similar to an EGM.

In this sense, many of the risk factors for problem gambling associated with Internet gambling may be heightened for gamblers who use mobile and supplementary devices. These include the convenience and easy accessibility and availability of gambling, enhanced privacy, perceived anonymity, and the reduced salience of electronic funds [75,76].

Finally, we observed that overall online gambling in adolescents, i.e. not taking into account the different modalities, compared with land-based gambling has higher rates of problematic gambling, gambling severity, and higher risk for problem gambling. Only one study [30] found none rates of problem gamblers among the online gamblers sample, but the prevalence of problematic behavior among mixed-mode gamblers (both land-based and online at the same time). Moreover, online gamblers were involved in a higher variety of gambling modes. In this sense, some authors have informed that more important that the specific form of gambling, is the involvement in multiple forms of gambling [77,78]. These results, jointly with the fact that adolescents with land-based gambling behavior used to utilize different gambling modes at the same time [79], suggest that online gambling is not only a specific gambling activity that can suppose a risk for developing health problems, but also it may be a combination of several gambling modes or a specific gambling use pattern. Furthermore, these patterns can change over time and may even be different in different cultural contexts.

Taking into account all the results, and in line with the mentioned literature, the association between problematic online gambling, consequences and gambling involvement may be influenced by the selected specific form of gambling, but this relationship is quite complex. On the one hand, it's possible that the inner characteristics of each form are associated with a higher probability of abuse. On the other hand, it could be that certain personality characteristics or motivations for gambling, are a risk to develop problems with gambling, and that these characteristics lead these types of adolescents to choose certain types of gambling games. Moreover, recent results pointed that the relationship between Internet gambling and problem gambling is complex and commonly mediated by engagement with land-based gambling [80].

In conclusion, consensus on the evaluation method of the problem and updated questionnaires collecting information regarding the new online betting modalities are critical; the relationships between specific forms of online gambling and related consequences are unclear, and future research needs to be focused not only how often gamblers engage in general, but also which specific games they play. Additionally, studies focused on clinical populations or performed in a more controlled setting are highly required.

\section{Limitations}

One of the limitations of our study refers to the restrictions in the language of the reviewed articles, and the possible heterogeneity naming Online Gambling in adolescents across the different studies. This can cause that some articles related to the topic could be missed. However, a search through the citations of the selected articles was carried out, and the abstracts (written in English) of articles in other languages were included in the review process. Another limitation is the limit of 45 first pages in Google Scholar, however, other authors have used this method before [81], without influence in the final result, and the search in other databases and in the citations of the reviewed articles may reduce the risk of bias.

\section{Author Contributions}

V.G-B and J.J.S. conceived and planned this article. V.G.-B. and J.J.S. carried out the search and revision of the literature. D.F. analyzed the data. V.G.-B. and J.J.S. drafted the study. All authors (V.G.-B., 
J.J.S., E.M., M.B., D.F., S.J.-M., A.d.P.-G. and J.R.) revised the article critically for important intellectual content. All authors (V.G.-B., J.J.S., E.M., M.B., D.F., S.J.-M., A.d.P.-G. and J.R.) commented on and approved the final manuscript and are accountable for all aspects of the work. All authors have read and agreed to the published version of the manuscript.

\section{Funding}

This work was funded by an AIS (Atención e Investigación en Socioaddiciones) intramural research program. This research is partially supported by the Marsden grant E2987-3648 (Royal Society of New Zealand) and by grant 2017 SGR 622 (GRBIO) from the Departament d'Economia i Coneixement de la Generalitat de Catalunya (Spain). These partial funders had no role in the study design, data collection, and analysis, decision to publish, or preparation of the manuscript.

\section{References}

1. Lorains FK, Cowlishaw S, Thomas SA. Prevalence of comorbid disorders in problem and pathological gambling: Systematic review and meta-analysis of population surveys. Addiction. John Wiley \& Sons Ltd. 2011; 106: 490-498.

2. Adolphe A, Khatib L, van Golde C, Gainsbury SM, Blaszczynski A. Crime and Gambling Disorders: A Systematic Review. J Gambl Stud. 2019; 35: 395-414.

3. Riley BJ, Harvey P, Crisp BR, Battersby M, Lawn S. Gambling-related harm as reported by concerned significant others: a systematic review and metasynthesis of empirical studies. J Fam Stud. 2018.

4. Ronzitti S, Soldini E, Lutri V, Smith N, Clerici M, Bowden-Jones H. Types of gambling and levels of harm: A UK study to assess severity of presentation in a treatment-seeking population in: Journal of Behavioral Addiction. J Behav Addict. 2016; 5: 439-447.

5. Dowling N, Smith $D$, Thomas T. Electronic gaming machines: Are they the "crack-cocaine" of gambling? Addiction. John Wiley \& Sons Ltd. 2005; 100: 33-45.

6. Christina Thomas A, Bates G, Moore S, Kyrios M, Meredyth D, Jessop G Gambling and the Multidimensionality of Accessibility: More Than Just Proximity to Venues. 2009.

7. Finlay K, Marmurek HHC, Kanetkar V, Londerville J. Casino Decor Effects on Gambling Emotions and Intentions. Environ Behav. 2010; 42: 524-545.

8. Abarbanel B, Bernhard B, Singh AK, Lucas A. Impact of virtual atmospherics and functional qualities on the online gambler's experience. Behav Inf Technol. 2015; 34: 1005-1021.

9. LaPlante DA, Nelson SE, LaBrie RA, Shaffer HJ. Men \& women playing games: Gender and the gambling preferences of lowa Gambling Treatment Program participants. J Gambl Stud. 2006; 22: 65-80.

10. Young M, Stevens M. Player preferences and social harm: An analysis of the relationships between player characteristics, gambling modes, and problem gambling. Int J Ment Health Addict. 2009; 7: 262-279.

11. Oliveira MPMT, Silva MTA. A Comparison of Horse-Race, Bingo, and Video Poker Gamblers in Brazilian Gambling Settings. J Gambl Stud. 2001; 17: 137-149.

12. Petry NM. A comparison of treatment-seeking pathological gamblers based on preferred gambling activity. Addiction. 2003; 98: 645-655.

13. Hing N, Russell AM, Browne M. Risk Factors for Gambling Problems on Online Electronic Gaming Machines, Race Betting and Sports Betting. Front Psychol. 2017; 8: 779.

14. Clarke D, Pulford J, Bellringer M, Abbott M, Hodgins DC. An Exploratory Study of Problem Gambling on Casino Versus Non-casino Electronic Gaming Machines. Int J Ment Health Addict. 2012; 10: 107-121.

15. Ricijas N, Dodig Hundric D, Huic A. Predictors of adverse gambling related consequences among adolescent boys. Child Youth Serv Rev. 2016; 67: 168-176.

16. McCormack A, Griffiths MD. Scoping study of the structural and situational characteristics of internet gambling. Int J Cyber Behav Psychol Learn. 2013; 3: 29-49.

17. McCormack A, Griffiths MD. Motivating and Inhibiting Factors in Online Gambling Behaviour: A Grounded Theory Study. Int J Ment Health Addict. 2012; 10: 39-53.

18. Zaman B, Geurden K, De Cock R, De Schutter B, Vanden Abeele V. Motivation profiles of online Poker players and the role of interface preferences: A laddering study among amateur and (semi-) professionals. Comput Human Behav. 2014; 39: 154-164.

19. Manzin M, Biloslavo R. Online gambling: Today's possibilities and tomorrow's opportunities. Manag Glob Transitions. 2008; 6: 95-110.

20. Hing N, Sproston K, Brook K, Brading R. The Structural Features of Sports and Race Betting Inducements: Issues for Harm Minimisation and Consumer Protection. J Gambl Stud. 2017; 33: 685-704.

21. Parke A, Griffiths MD. Identifying risk and mitigating gambling-related harm in online poker. Journal of Risk Research. Routledge. 2018; 21: 269-289.

22. Sapthiang S, Van Gordon W, Shonin E, Griffiths MD. Adolescent problem gambling requires community-level health promotion approaches. Addiction Research and Theory. Taylor and Francis Ltd. 2020; 28: 91-94.

23. Blinn-Pike L, Worthy SL, Jonkman JN. Adolescent gambling: a review of an emerging field of research. J Adolesc Health. 2010; 47: 223-236.

24. Ariyabuddhiphongs V. Adolescent Gambling: A Narrative Review of Behavior and Its Predictors. Int J Ment Health Addict. 2013; 11: 97-109.

25. Gupta R, Derevensky J. Understanding the etiology of youth problem gambling. In: J Derevensky, D Shek \& JM (Eds, editor. Youth gambling problems: The hidden addiction. Berlin: Berlin: De Gruyter. 2011.

26. Geidne S, Froding K, Larsson M. Gambling among adolescents with and without hearing loss. Asian J Gambl Issues Public Heal. 2016; 6: 8.

27. Weinberger AH, Franco CA, Hoff RA, Pilver C, Steinberg MA, Rugle L, et al. Cigarette smoking, problem-gambling severity, and health behaviors in highschool students. Addict Behav Reports. 2015; 1: 40-48.

28. Foster DW, Hoff RA, Pilver CE, Yau YHC, Steinberg MA, Wampler J, et al. Correlates of gambling on high-school grounds. Addict Behav. 2015; 51: 5764.

29. Calado F, Alexandre J, Griffiths MD. Prevalence of Adolescent Problem Gambling: A Systematic Review of Recent Research. Journal of Gambling Studies. Springer New York LLC. 2017; 33: 397-424.

30. Gonzalez-Roz A, Fernandez-Hermida JR, Weidberg S, Martínez-Loredo V, Secades-Villa R. Prevalence of Problem Gambling Among Adolescents: A Comparison Across Modes of Access, Gambling Activities, and Levels of Severity. J Gambl Stud. 2017; 33: 371-382.

31. Choliz M, Lamas J. "Hagan juego, menores!" Frecuencia de juego en menores de edad y su relación con indicadores de adicción al juego. "Place your bets, children!" The frequency of gambling among minors and their relationship with gambling addiction indicators. Rev Española Drog. 2017; 42: 34-47.

32. Jackson AC, Dowling N, Thomas SA, Bond L, Patton G. Adolescent gambling behaviour and attitudes: A prevalence study and correlates in an Australian population. Int J Ment Health Addict. 2008; 6: 325-352.

33. Splevins K, Mireskandari S, Clayton K, Blaszczynski A. Prevalence of adolescent problem gambling, related harms and help-seeking behaviours among an Australian population. J Gambl Stud. 2010; 26: 189-204.

34. Yip SW, Desai RA, Steinberg MA, Rugle L, Cavallo DA, Krishnan-Sarin S, et al. Health/functioning characteristics, gambling behaviors, and gamblingrelated motivations in adolescents stratified by gambling problem severity: Findings from a high school survey. Am J Addict. 2011; 20: 495-508.

35. Messerlian C, Derevensky JL. Youth gambling: A public health perspective. J Gambl Issues. 2005: 14. 
36. Potenza MN. The neurobiology of pathological gambling and drug addiction: An overview and new findings. In: Philosophical Transactions of the Royal Society B: Biological Sciences. Royal Society. 2008; 363: 3181-3189.

37. Gainsbury MS, Hing N, Delfabbro PH, King DL. A taxonomy of gambling and casino games via social media and online technologies. Int Gambl Stud. 2014; 14: 196-213.

38. Aricak OT. Problematic online betting among Turkish adolescents. J Gamb Stud. 2019; 35: 31-45.

39. Baggio S, Dupuis M, Berchtold A, Spilka S, Simon O, Studer J. Is gambling involvement a confounding variable for the relationship between interne gambling and gambling problem severity? Comput Human Behav. 2017; 71 148-152.

40. Calado F, Alexandre J, Griffiths MD. How coping styles, cognitive distortions and attachment predict problem gambling among adolescents and young adults. J Behav Addict. 2017; 6: 648-657.

41. Canale N, Griffiths MD, Vieno A, Siciliano V, Molinaro S. Impact of Internet gambling on problem gambling among adolescents in Italy: Findings from a large-scale nationally representative survey. Comput Human Behav. 2016 57: 99-106.

42. Tara EM, Leatherdale ST, Turner NE. An examination of internet and landbased gambling among adolescents in three Canadian provinces: Results from the youth gambling survey (YGS). BMC Public Health. 2016; 16.

43. Fu W, Yu CKC. Predicting Disordered Gambling with Illusory Control, Gaming Preferences, and Internet Gaming Addiction among Chinese Youth [Internet] Vol. 13, International Journal of Mental Health and Addiction. Springer New York LLC. 2015; 13: 391-401.

44. King DL, Delfabbro PH, Kaptsis D, Zwaans T. Adolescent simulated gambling via digital and social media: An emerging problem. Comput Human Behav. 2014: 31: 305-313.

45. King DL, Delfabbro PH. Adolescents' perceptions of parental influences on commercial and simulated gambling activities. Int Gambl Stud. 2016; 16: 424 441.

46. Kristiansen SG, Jensen SM. Prevalence and correlates of problematic gambling among Danish adolescents. Int J Soc Welf. 2014; 23: 89-99.

47. Lupu V, Todirita IR. Updates of the Prevalence of Problem Gambling in Romanian Teenagers. J Gambl Stud. 2013; 29: 29-36.

48. Veselka L, Wijesingha R, Leatherdale ST, Turner NE, Elton-Marshall $T$. Factors associated with social casino gaming among adolescents across game types. BMC Public Health. 2018; 18: 1167

49. Weidberg S, Gonzalez-Roz A, Fernandez-Hermida JR, Martinez-Loredo V, Grande-Gonsende A, Garcia-Perez, et al. Gender differences among adolescent gamblers. Pers Individ Dif. 2018; 125: 38-43.

50. Wong ILK, So EMT. Internet gambling among high school students in Hong Kong. J Gambl Stud. 2014; 30: 565-576.

51. Zhao Y, Marchica L, Derevensky JL, Ivoska W. Mobile gambling among youth: A warning sign for problem gambling? J Gambl Issues. 2018; 38: 268 282.

52. Hollen L, Dorner R, Griffiths MD, Emond A. Gambling in Young Adults Aged 17-24 Years: A Population-Based Study. J Gambl Stud. 2020.

53. Rossow I, Hansen MB, Storvoll EE. Changes in youth gambling after the removal of slot machines in Norway. Nord Stud Alcohol Drugs. 2013; 30 317-330.

54. Winters KC, Stinchfield RD, Fulkerson J. Toward the development of an adolescent gambling problem severity scale. J Gambl Stud. 1993; 9: 63-84.

55. APA. Diagnostic and Statistical Manual of Mental Disorders, $5^{\text {th }}$. Edition (DSM-5). American Psychiatric Association, editor. Washington DC. 2013.

56. Fisher S. Developing the DSM-IV-DSM-IV Criteria to Identify Adolescent Problem Gambling in Non-Clinical Populations. J Gambl Stud. 2000; 16: 253 273
57. APA. Diagnostic and Statistical Manual of Mental Disorders, $4^{\text {th }}$ Edition (DSMIV). American Psychiatric Association, editor. Washington DC. 1994

58. Tremblay J, Stinchfield R, Wiebe J, Wynne H. Canadian Adolescent Gambling Inventory (CAGI) Phase III Final Report. 2010.

59. Ferris J, Wynne HJ. The Canadian problem-gambling index: Final report. Ottawa: Canadian Centre on Substance Abuse. 2001

60. Gamblers Anonymous. The 20 questions of the Gamblers Anonymous American Association. $3^{\text {rd }}$ ed. Los Angeles, CA: Gamblers Anonymous Publishing. 1984

61. Toce-Gerstein M, Gerstein DR, Volberg RA. The NODS-CLiP: A rapid screen for adult pathological and problem gambling. J Gambl Stud. 2009; 25: 541555.

62. Baggio S, Gainsbury SM, Starcevic V, Richard JB, Beck F, Billieux J. Gende differences in gambling preferences and problem gambling: a network-level analysis. Int Gambl Stud. 2018; 18: 512-525.

63. Scalese M, Bastiani L, Salvadori S, Gori M, Lewis I, Jarre P, et al. Association of Problem Gambling with Type of Gambling Among Italian General Population. J Gambl Stud. 2016; 32: 1017-1026.

64. Delfabbro P, King DL, Derevensky JL. Adolescent Gambling and Problem Gambling: Prevalence, Current Issues, and Concerns. Current Addiction Reports. Springer; 2016; 3: 268-274.

65. Meyer G, Fiebig M, Hafeli J, Morsen C. Development of an assessment too to evaluate the risk potential of different gambling types. Int Gambl Stud. 2011; 11: 221-236.

66. Pisarska A, Ostaszewski K. Factors associated with youth gambling: longitudinal study among high school students. Public Health. 2020.

67. Husky MM, Michel G, Richard JB, Guignard R, Beck F. Gender differences in the associations of gambling activities and suicidal behaviors with problem gambling in a nationally representative French sample. Addict Behav. 2015; 45: $45-50$.

68. Ronzitti S, Kraus SW, Hoff RA, Clerici M, Potenza MN. Problem-gambling severity, suicidality and DSM-IV Axis II personality disorders. Addict Behav. 2018; 82: 142-150.

69. Shead NW, Derevensky JL, Gupta R. Risk and protective factors associated with youth problem gambling. Int J Adolesc Med Health. 2010; 22: 39-58.

70. Volberg R, Gupta R, Griffiths M, Olason D, Delfabbro P. An international perspective on youth gambling prevalence studies. Int J Adolesc Med Health. 2010; 22: 3-38

71. Nower L, Blaszczynski A. A pathways approach to treating youth gamblers. In: Gambling Problems in Youth: Theoretical and Applied Perspectives [Internet]. Springer US. 2005; 189-209.

72. Breen RB, Zimmerman M. Rapid onset of pathological gambling in machine gamblers. J Gambl Stud. 2002; 18: 31-43.

73. Breen RB. Rapid onset of pathological gambling in machine gamblers: A replication. eCOMMUNITY Int J Ment Heal Addict. 2004; 2.

74. Stea JN, Hodgins DC, Fung T. Abstinence versus moderation goals in brief motivational treatment for pathological gambling. J Gambl Stud. 2015; 31: 1029-1045.

75. Gainsbury S, Wood R, Russell A, Hing N, Blaszczynski A. A digital revolution: Comparison of demographic profiles, attitudes and gambling behavior of Internet and non-Internet gamblers. Comput Human Behav. 2012; 28: 13881398.

76. MacKay TL, Hodgins DC. Cognitive distortions as a problem gambling risk factor in Internet gambling. Int Gambl Stud. 2012; 12: 163-175.

77. LaPlante DA, Afifi TO, Shaffer HJ. Games and Gambling Involvement among Casino Patrons. J Gambl Stud. 2013; 29: 191-203.

78. LaPlante DA, Nelson SE, Gray HM. Breadth and depth involvement: Understanding Internet gambling involvement and its relationship to gambling problems. Psychol Addict Behav. 2014; 28: 396-403. 
79. Welte JW, Barnes GM, Tidwell MCO, Hoffman JH. The Association of Form of Gambling With Problem Gambling Among American Youth. Psychol Addict Behav. 2009; 23: 105-112.

80. Gainsbury SM, Angus DJ, Blaszczynski A. Isolating the impact of specific gambling activities and modes on problem gambling and psychological distress in internet gamblers. BMC Public Health. 2019; 19: 1372.
81. Kaptsis D, King D, Delfabbro P, Gradisar M. Withdrawal symptoms in internet gaming disorder: A systematic review. Clin Psychol Rev. 2016; 43: 58-66. 